\title{
Editorial
}

\section{Decreased body fat and gastric cancer: does the hen come before the egg, or vice versa?}

\author{
Peter Gunvén \\ Department of Oncology, Karolinska University Hospital, SE 17176 Stockholm, Sweden
}

About 13000 scientific articles concern fat and cancer. Gastric cancer is the subject of only a few hundred of them, however, despite its high global prevalence and the nutritional consequences of this disease and its therapy. A frequent theme is epidemiology, e.g., the associations between obesity and steroid hormonerelated cancers or between BMI-related gastroesophageal reflux and cancers of the gastroesophageal junction, as recently reviewed [1]. Another topic concerns changes in body fat mass due to tumor- or therapy-induced malnutrition.

In this issue of Gastric Cancer, Otani and co-workers [2] describe their study in early gastric cancer (EGC) of another aspect of fat distribution. They quantified the total, subcutaneous, and visceral fat masses on preoperative computed tomography (CT) scans for diagnostic purposes. The selection of EGC was intended to avoid the nutritional consequences of advanced cancer, and subgroups of EGC were compared with each other and with colon cancer patients as well as with the Japanese population.

The main finding was that patients of both sexes with undifferentiated EGC invading the submucosa, and females with undifferentiated mucosal EGC, had smaller volumes of visceral and subcutaneous fat than patients with differentiated tumors or patients with colorectal cancer. The authors speculate that subcutaneous fat may have a closer relation to EGC than visceral fat, and that the more consistent (in both $\mathrm{m}$ and sm cancers) difference in fat volumes between undifferentiated and differentiated EGC patients in females could be due to the greater amounts of subcutaneous fat in women.

The findings cannot be explained easily with today's knowledge. Were they simply due to random variation

Offprint requests to: P. Gunvén and reported after publication bias? Or do they reflect a possible influence of existing cancer or of a causative Helicobacter pylori infection on nutritional status, or suggest unknown pathogenetic mechanisms for EGC?

A large number of comparisons were performed. Four partially interrelated parameters, i.e., total, visceral, and subcutaneous fat areas and the ratio of visceral to subcutaneous fat, were studied in EGC versus colon cancer and compared also with data from the Japanese population. EGC was subgrouped according to differentiation, depth of invasion, location, H. pylori positivity, presence of ulceration, symptomatology, and in patient categories defined by sex and age.

Occasionally the compared subsets were defined by several of these variables, such as male versus female subjects in relation to age and differentiation, giving eight patient groups in each of which four parameters were studied resulting in 32 measurements. We would not be surprised to find a statistically significant difference between some of these measurements due to chance.

Against this interpretation speak, however, the more or less consistent reductions of fat volumes in many subsets of undifferentiated cancers, which were defined by unrelated variables.

A selection bias must be discussed since the authors report that only 210 out of 550 patients with EGC had preoperative CT scans. A selection for diagnostic radiology of symptomatic or weight-losing patients seems possible. However, at most $20 \%$ of the patients under study were symptomatic, and a slightly higher proportion had an active ulcer. These factors did not influence the fat volumes, which seems to argue against the presence of a selection bias and against a change in fat deposition secondary to tumor symptoms. Neither diabetes nor $H$. pylori infection-the latter, however, only being assessed in a minority of patients-influenced the degree of tumor differentiation. 
If we favor the authors' hypothesis that the differences are real and that they suggest pathogenetic mechanisms starting with changes in the fat distribution, in which direction should we speculate? Only a few tumors were localized in the gastroesophageal junction and classified as Siewert's type II, and they did not differ from other tumors with regard to fat distribution. A simple mechanistic chain of obesity-refluxcarcinogenesis was thus not supported. The authors therefore seem to favor alternative mechanisms.

Recently, adipose tissue has become considered as more than a storage of calories and instead characterized as a major endocrine organ [3]. As the authors discuss, it secretes adipokines and adipose-derived hormones and mediators, substances such as adiponectin, leptin, resistin, and others.

The substances seem, among a variety of functions, to influence inflammation and angiogenesis, to have cytoprotective effects on gastric mucosa, and possibly to affect cancer cells directly. For example, recent studies suggest that the levels of some such substances in both serum and white adipose tissue in the mesentery relate to inflammatory bowel disease. The substances could therefore be speculated to interact with the so-called Correa cascade of gastritis-atrophymetaplasia-dysplasia-gastric cancer. In fact, low adiponectin serum levels were found in Japanese patients with gastric cancer, and these levels related to tumor characteristics in undifferentiated but not in differentiated cancers [4]. In addition to antiangiogenic properties, a direct antitumor effect was suggested by the presence of adiponectin receptors on cultured gastric cancer cells and induction of apoptosis in such cells by adiponectin [5].

Leptin in serum comes mainly from adipose tissue, but the substance is found also in gastric mucosa, even though opinions have differed on whether its mRNA is present there. Also leptin and its receptor were demonstrated in gastric cancer, with significantly lower expression of leptin in poorly differentiated forms [6], and leptin was reported to stimulate proliferation of gastric cancer cells. The presence of ligands, adiponectin and leptin, as well as their receptors in the stomach, suggests the possibility of auto- or paracrine loops, even though a majority of the adipokines are produced in adipose tissue.

A further finding of possible relevance is that successful vaccination against Helicobacter in an animal system related to upregulation of genes for adiponectin and resistin [7]. A complex network of interactions between various factors secreted by adipose tissue or locally in the stomach, host responses against, e.g., the carcinogen $H$. pylori, protection of gastric mucosal cells, tumor differentiation, and vascularization may therefore be assumed to exist, but its details have to be clarified.

Otani and co-workers are to be commended for their hypothesis-generating study despite its methodological problems. Their continued work will concern adipokines in relation to the development and progression of gastric cancer and may, together with the work of other groups, improve the present lack of knowledge.

\section{References}

1. Renehan AG, Tyson M, Egger M, Heller RF, Zwahlen M. Bodymass index and incidence of cancer: a systematic review and metaanalysis of prospective observational studies. Lancet 2008;371: 569-78.

2. Otani K, Kitayama J, Kaisaki S, Ishigami H, Hidemura A, Fujishiro $\mathrm{M}$, et al. Early gastric cancer shows different associations with adipose tissue volume depending on histological type. Gastric Cancer 2008;11:86-95.

3. Rondinone CM. Adipocyte-derived hormones, cytokines, and mediators. Endocrine 2006;29:81-90.

4. Ishikawa M, Kitayama J, Kazama S, Hiramatsu T, Hatano K, Nagawa H. Plasma adiponectin and gastric cancer. Clin Cancer Res. 2005;11:466-72.

5. Ishikawa M, Kitayama J, Yamauchi T, Kadowaki T, Maki T, Miyato $\mathrm{H}$, et al. Adiponectin inhibits the growth and peritoneal metastasis of gastric cancer through its specific membrane receptors AdipoR1 and AdipoR2. Cancer Sci. 2007;98:1120-7. Epub 2007 Apr 23.

6. Hong SJ, Kwon KW, Kim SG, Ko BM, Ryu CB, Kim YS, et al. Variation in expression of gastric leptin according to differentiation and growth pattern in gastric adenocarcinoma. Cytokine 2006;33:66-71. Epub 2006 Jan 23.

7. Mueller A, O'Rourke J, Chu P, Kim CC, Sutton P, Lee A, et al. Protective immunity against Helicobacter is characterized by a unique transcriptional signature. Proc Natl Acad Sci U S A. 2003;100:12289-94. Epub 2003 Oct 3. 